\title{
Recommendations on quantities, symbols and measurement units for publications in ACQUAL: the correct use of equations for calculating chemical measurement results
}

\author{
Wolfgang Richter
}

Received: 19 August 2008/ Accepted: 27 August 2008/Published online: 2 October 2008

(C) Springer-Verlag 2008

Chemical measurement results are usually not obtained directly, but are calculated from input quantities using equations which describe the measurement (model or measurement equations). As a simple example, the measurement of the mass concentration of lead in a waste water sample is considered. The basic equation is:

$\gamma(\mathrm{Pb})=m(\mathrm{~Pb}) / V \quad$ or in short $\quad \gamma=m / V$

where $V$ is the sample volume and $\gamma$ is the preferred symbol for the mass concentration in the new edition of "Quantities, Units and Symbols in Physical Chemistry" (IUPAC Green Book, 3rd edn, 2007, subclause 2.10). The mass of lead, $m(\mathrm{~Pb})$, contained in this volume is assumed to be obtained using an appropriate analytical method, the details of which are not of interest here. Without further information, one would expect the result to be in the measurement unit $\mathrm{g} / \mathrm{L}$ (or, expressed in base units, in $\mathrm{kg} / \mathrm{m}^{3}$ ).

In analytical practice, the mass concentration is frequently expressed in $\mathrm{mg} / \mathrm{L}$. In order to obtain the result in this special unit, analysts sometimes tend to the following habit (accordingly applied to this example): They introduce a "conversion factor" of 1000 into the equation, resulting in:

$\gamma(\mathrm{Pb})=1000 m(\mathrm{~Pb}) / V$

and add the information that $m$ is the mass in $g$ and $V$ is the volume in L.

Equation 2 is obviously in contradiction to Eq. 1 and cannot be correct as it is written. What is intended to be said with Eq. 2 together with the sentence following it can be correctly expressed by applying the rules of quantity

W. Richter $(\square)$

Stadeweg 1, 38106 Braunschweig, Germany

e-mail: wolfgang.k.richter@t-online.de calculus, the algebra of quantities (IUPAC Green Book, 3rd edn, 2007, subclauses 1.1 and 7.1), resulting in:

$\frac{\gamma}{\mathrm{mg} / \mathrm{L}}=1000 \frac{\mathrm{m} / \mathrm{g}}{\mathrm{V} / \mathrm{L}}$.

Here, use is made of the special set of units chosen by the analyst and of the definition that the value of a physical quantity is the product of a numerical value and a unit. Equation 2 does not follow this definition, since quantity symbols are written while their numerical values only, appearing as quotients of the quantity values and their units in Eq. 3, are meant.

Another way of writing Eq. 2 correctly is:

$\{\gamma\}_{\mathrm{mg} / \mathrm{L}}=1000\{m\}_{\mathrm{g}} /\{V\}_{\mathrm{L}}$

where the symbols in curly brackets are the numerical values of the quantities in the indicated units.

Such equations between numerical values (numerical value equations) are not recommended, since they are valid only for the special choice of units. Equations like Eq. 2, in which quantity symbols are used for numerical values, are not acceptable.

Quantity equations like Eq. 1 should always be preferred (ISO 31-0, subclause 2.2.2; see also ISO/IEC Guide 99-12:2007 "International Vocabulary of MetrologyBasic and General Concepts and Associated Terms" (VIM), 3rd edn, subclause 1.21, available as JCGM 200:2008 at http://www.bipm.org/en/publications/guides/vim). Quantity equations have the advantage that their validity is independent of the choice of units. The result can finally be converted to any unit desired. If numerical value equations are required in special cases, the units chosen shall be clearly stated, for example, in the ways as shown in Eqs. 3 and 4. 